\title{
Network structure dependence on unconstrained isothermal-recovery processes for shape-memory thiol-epoxy "click" systems
}

Alberto Belmonte ${ }^{1}$ Xavier Fernández-Francos ${ }^{2}$ Silvia De la Flor ${ }^{1}$ Àngels Serra ${ }^{3}$

1. Department of Mechanical Engineering. Universitat Rovira i Virgili. Av. Països Catalans 26, 43007 Tarragona, Spain.

2. Thermodynamics Laboratory, ETSEIB, Universitat Politècnica de Catalunya. Av. Diagonal 647, 08028 Barcelona, Spain.

3. Department of Analytical and Organic Chemistry, Universitat Rovira i Virgili, C/Marcel/lí Domingo s/n, 43007, Tarragona, Spain;

Corresponding author: e-mail: silvia.delaflor@urv.net

\begin{abstract}
The shape-memory response (SMR) of "click" thiol-epoxy polymers produced using latent catalysts, with different network structure and thermo-mechanical properties, was tested on unconstrained shape-recovery processes under isothermal conditions. Experiments at several programming temperatures $\left(T_{\text {prog }}\right)$ and isothermal-recovery temperatures $\left(T_{\text {iso }}\right)$ were carried out and the shape-memory stability wasere analysed through various consecutive shape-memory cycles. The temperature profile during the isothermal-recovery experiments was monitored, and it showed that the shape-recovery process takes place while the sample is becoming thermally stable and before stable isothermal temperature conditions are eventually reached. The shaperecovery process takes place in two different stages regardless of $T_{\text {iso: }}$ a slow initial stage until the process is triggered at a temperature strongly related with the beginning of network relaxation, followed by the typical exponential decay of the relaxation processes until completion at a temperature below or very close to $T_{g}$. The shape-recovery process is slower in materials with more densely crosslinked and hindered network structures. The shape-recovery time $\left(t_{s r}\right)$ is significantly reduced when the isothermal-recovery temperature $T_{i s o}$ increases from below to above $T_{g}$ because the network relaxation dynamics accelerates. However, the temperature range from the beginning to the end of the recovery process is hardly affected by $T_{\text {iso; }}$; at higher $T_{\text {iso }}$ it is only slightly shifted to higher temperatures. These results suggest that the shape-recovery process can be controlled by changing the network structure and working at $T_{i s o}<T_{g}$ to maximize the effect of the structure and/or by increasing $T_{\text {iso }}$ to minimize the effect but increasing the shape-recovery rate.
\end{abstract}

Keywords Thiol-epoxy; shape-memory polymer; isothermal-recovery; click chemistry

\section{Introduction}

Shape-memory polymers $(S M P)$ are materials that can retain a temporary shape and recover their original shape through an external stimulus which depends on the physical nature of the polymer (Hager et al. 2015; Scalet et al. 2015; Zhao et al. 2015). Temperature is one of the most common stimuli for the shape-memory effect- because it either relaxes the network or melts one of the components, which leads to the recovery of the original shape (Lendlein and Sauter 2013; Sun et al. 2012). Recently, the increasing demand for smart materials in aerospace 
engineering, electronic devices and structural applications has redirected the focus of a great deal of research to thermoset-based SMPs (Ebara 2015; Leng et al. 2015; Song et al. 2015; Tandon et al. 2016). Thermoset-based SMPS are more thermally stable, stronger and more durable than thermoplastic-based SMPs. They also fix the temporary shape better (almost 100\%), have higher shape-recovery rates and can perform mechanical work (Arrieta et al. 2014a; Arrieta et al. 2014b; Lakhera et al. 2012; Santiago et al. 2016). Of all the different types, epoxy-based SMPs are worthy of special mention because of their excellent mechanical, electrical, optical and thermal properties (Santhosh Kumar et al. 2013, Tandon et al. 2012). However, the rigid structure and fragility of common epoxy thermosets means that they are less deformable and less applicable. One way of overcoming this limitation is to use curing agents with long, aliphatic chains (Feldkamp and Rousseau 2011; Leonardi et al. 2011). This can significantly enhance the deformability of the materials, but it reduces the glass transition temperature $\left(T_{g}\right)$ quite considerably and may worsen the strength. A different strategy is to program the temporary shape at the onset point of the glass transition process $\left(T_{g}{ }^{\prime}\right)$ measured by DMA (Feldkamp and Rousseau 2010; Yakacki et al. 2008b), thereby increasing the deformability three-to-five-_fold and enhancing the mechanical strength of the material. Therefore, by properly combining curing agents and programming conditions, both the deformability and strength can be improved. Recently, Santiago et al. (Santiago et al. 2015) reported SMPs with enhanced strength in comparison with other works (Fan et al. 2013; Feldkamp and Rousseau 2011; Leonardi et al. 2011), when hyperbranched poly(ethyleneimine) polymers were combined with simple monomeric aliphatic amine curing agents reacted with an epoxy resin. In a previous study (Belmonte et al. 2015), we developed thiolepoxy materials with tailored network structures and, in combination with optimal programming conditions at $T_{g}{ }^{\prime}$, the strain and stress at break were as high as $96 \%$ and $55 \mathrm{MPa}$.

"Click" chemistry makes it possible to develop materials easily and efficiently (Binder and Sachsenhofer 2007). Of all the various "click" systems, the thiolepoxy mechanism is particularly interesting because hydroxyl and thioether groups can be formed in a single step and then further transformed into other polymeric structures (Carlborg et al. 2014; Flores et al. 2013). Tertiary amines are commonly used to catalyzse the thiol-epoxy reaction (Jin et al. 2015; Loureiro et
Formatted: Not Highlight

Formatted: Not Highlight

Formatted: Not Highlight 
al. 2015) but they are usually too reactive to prepare stable one-pot formulations. Latent catalysts such as encapsulated imidazoles or urones have been shown to significantly increase the pot-life of thiol-epoxy formulations allowing an easily handling and processing of the reactive mixture (Brändle and Khan 2012; Guzmán et al. 2014). Another advantage of using latent initiators is that the curing process occurs rapidly at a higher temperature and leads to materials with welldefined network structures and enhanced mechanical properties (Berg et al. 2014).

Nevertheless, since the material and the programming process are optimized to obtain the desired thermal and mechanical properties, the shape-recovery process is inherently modified by changes in the network structure. Therefore, it is of key importance to establish a well-defined relation between the network structure and the expected shape-recovery process. Understanding these relations is essential in structural applications in which the control and the stability of the shape-recovery process are of utmost importance in defining how the material will be applied. As is well-known, all viscoelastic materials follow a time_-temperature relation during relaxation processes which depends on their network structure. The shaperecovery process occurs during network relaxation. Although it is widely acknowledged that, in unconstrained experiments, network relaxation dynamics are more important to the shape-recovery process than other network parameters such as crosslinking density and modulus decrease (from the glassy to the rubbery state) (Yakacki et al. 2008a; Yakacki et al. 2007), further studies are required if this is to be confirmed (Chen and Nguyen 2011; Pandini et al. 2013; Rousseau and Xie 2009).

Following our previous work on the characterization of thiol-epoxy materials with shape-memory properties (Belmonte et al. 2015), here we focus on how the network structure affects unconstrained shape-recovery processes under isothermal conditions. Materials with different network structures and therefore different transition temperatures and mechanical properties are obtained by combining epoxy monomers and thiol crosslinkers with different functionalities. The materials are programmed at $T_{g}{ }^{\prime}$ but tested at different isothermal-recovery temperatures $\left(T_{i s o}\right)$ with the purpose of elucidating the network structure-shaperecovery relation. By analysing the time--temperature dependence on the shaperecovery process, we expect to identify and highlight the parameters of the 
network structure that are most important to the shape-recovery process and define qualitative and quantitative criteria for the dependence so that tools can be designed for the operational design of SMPS.

\section{Materials and methods}

\subsection{Materials}

A commercial epoxy resin, diglycidyl ether of bisphenol A (DGEBA, GY240, Huntsman, Everberg, Belgium), with a molecular weight per epoxy equivalent unit of $182 \mathrm{~g} / \mathrm{eq}$ was used as the main epoxy resin. Pentaerythritol tetrakis(3mercaptopropionate) (4thiol), with a molecular weight per thiol equivalent unit of $122.17 \mathrm{~g} / \mathrm{eq}$ and trimethylolpropane tris(3-mercaptopropionate) (3thiol), with a molecular weight per thiol equivalent unit of $132.85 \mathrm{~g} / \mathrm{eq}$ (Sigma-Aldrich, St. Louis, MO, USA) were used separately as thiol crosslinking agents. In addition, tris(2,3-epoxypropyl)isocyanurate (iso), with a molecular weight per epoxy equivalent unit of $99.09 \mathrm{~g} / \mathrm{eq}$ (Sigma-Aldrich, St. Louis, MO, USA) was used as a modifier (30:70\%wt. iso:DGEBA), while keeping the 1:1 stoichiometric ratio between epoxy and thiol groups in the formulations. An encapsulated imidazole (LC80, AC Catalysts) was used as a latent initiator in a quantity of 0.5 parts of catalyst per hundred parts of epoxy resin (phr). The composition of the different formulations considered isare listed in Table 1 .

The formulations were prepared by manually stirring the components in a glass vial and carefully pouring the mixtures into an open Teflon mold. The curing process was carried out in an oven for one hour at $120^{\circ} \mathrm{C}$ followed by one hour at $150^{\circ} \mathrm{C}$ to ensure that the process was completed. Scheme 1 shows the expected network for the mixtures without $i \mathrm{so}_{2}$ and Scheme 2 presents the changes expected in mixtures with iso (the crosslinking points have been highlighted).
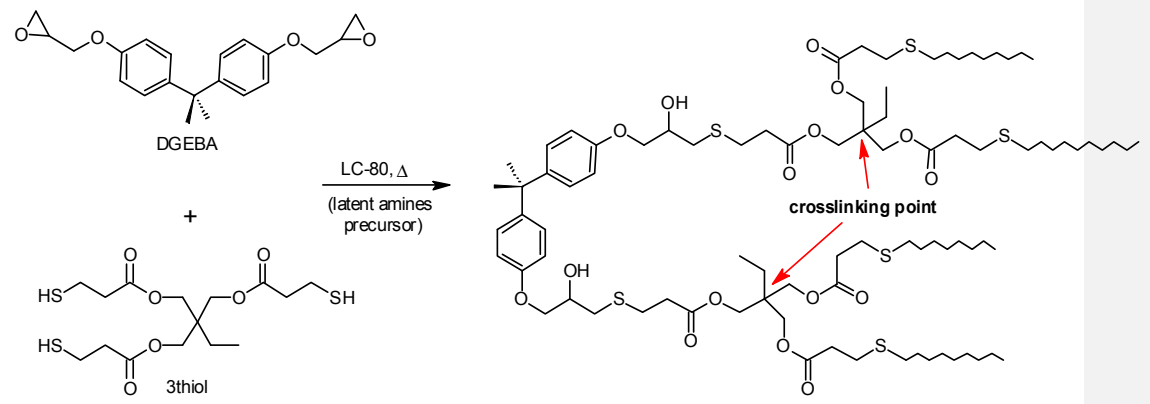
Scheme 1. Reaction scheme and expected network structure using DGEBA as epoxy resin, 3thiol as curing agent, and LC-80 as initiator (3thiol-DGEBA-LC80)

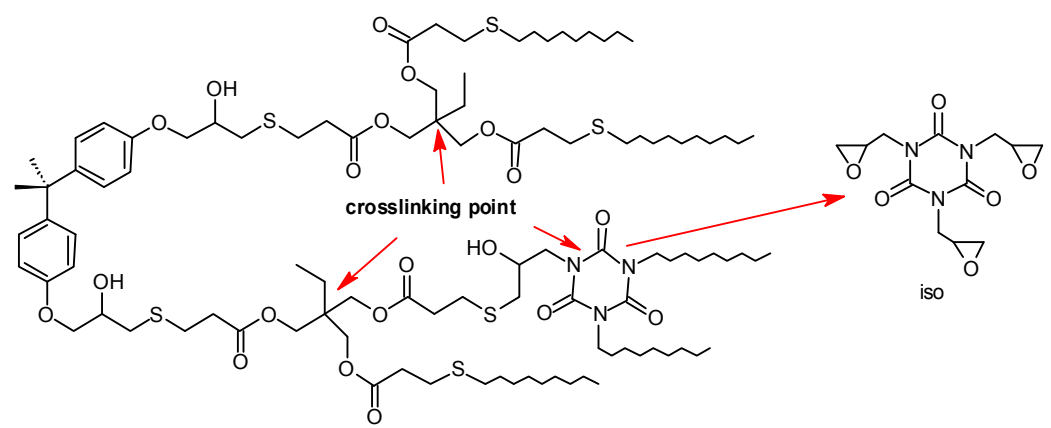

Scheme 2. Expected network structure of the 3thiol-DGEBA-LC80 formulation modified with the iso component

Table 1. Composition of the different formulations of study

\begin{tabular}{ccccc}
\hline Formulation & $\begin{array}{c}\text { DGEBA } \\
\text { (wt.\%) }\end{array}$ & $\begin{array}{c}\text { thiol } \\
\text { (wt.\%) }\end{array}$ & $\begin{array}{c}\text { iso } \\
\text { (wt.\%) }\end{array}$ & $\begin{array}{c}\text { LC80 } \\
\text { (wt.\%) }\end{array}$ \\
\hline 3thiol-NEAT & 57.64 & 42.07 & 0.00 & 0.29 \\
3thiol-(30\%)iso & 36.49 & 47.61 & 15.64 & 0.26 \\
\hline 4thiol-NEAT & 59.66 & 40.04 & 0.00 & 0.30 \\
4thiol-(30\%)iso & 37.95 & 45.52 & 16.26 & 0.27 \\
\hline
\end{tabular}

\subsection{Thermomechanical characterization}

A DMA Q800 (TA instrument) equipped with a single-cantilever $(10 \mathrm{~mm})$ clamp was used for the dynamic mechanical thermal analysis. The experiments were performed at a controlled heating rate $\left(3{ }^{\circ} \mathrm{C} / \mathrm{min}\right)$, from $30^{\circ} \mathrm{C}$ to $120^{\circ} \mathrm{C}$, at $1 \mathrm{~Hz}$ and $10 \mu \mathrm{m}$ of amplitude. The samples were thoroughly polished until they were shaped like a prismatic rectangle $\left(\approx 20 \times 7.5 \times 1.5 \mathrm{~mm}^{3}\right)$. The $T_{g}$ was determined by the peak of the $\tan \delta$ curve. The $T_{g}{ }^{E^{\prime}}$ was calculated as the onset point of the decrease in storage modulus due to the mechanical relaxation of the network. The width at half-height (FWHM) and the peak of the $\tan \delta$ curve were used as an indication of the heterogeneity of the relaxation process. An onset period of the network relaxation process was determined by integrating the $\tan \delta$ curve and calculating the temperature range $\left(\Delta T_{n e t}\right)\left({ }^{\circ} \mathrm{C}\right)$ between $0.1 \%$ andte $2 \%$ of the whole relaxation process $(100 \%$ being the end of the relaxation process). According to the Affine model for ideal rubbers (see equation (1)), the network strand density $\left(v_{e}\right)$ is related to the relaxed storage modulus $\left(E_{r}{ }^{\prime}\right)$ at low strain rates. The Affine model needs to be modified (see equation (2)) to account for the 
different functionality and fluctuation of the crosslinking points (a detailed explanation can be found in our previous study (Belmonte et al. 2015)):-

$$
\begin{aligned}
& E_{r}{ }^{\prime}=3 \cdot R \cdot T \cdot v_{e,} \\
& v_{e}=\sum_{f \geq 3} \frac{f-2}{2} \cdot n_{f}
\end{aligned}
$$

where $R$ is the universal gas constant, $T$ is the temperature at which the modulus $E_{r}{ }^{\prime}$ is calculated and $n_{f}$ is the density of crosslinking points with functionality $f$.

\subsection{Shape-memory response $(S M R)$}

Isothermal-recovery experiments were performed to analyse the SMR using the "U" bending test methodology. Prismatic rectangular shaped samples of $40 \times 7.5$ $\mathrm{x} 1.5 \mathrm{~mm}^{3}$ (length $\mathrm{x}$ width $\mathrm{x}$ thickness) were polished to flat parallel surfaces (original shape). The U-shaped programming process was performed as follows: the samples were heated up to the programming temperature and then deformed to the U-shape using the bending device shown in Fig.tre 1(a). Both the device and the sample were rapidly cooled using ice water while the stress was maintained for one minute. Afterwards, the stress was released and the U-shape was fixed. The shape-recovery process was performed by placing the programmed sample inside the preheated oven at an isothermal-recovery temperature $\left(T_{i s o}\right)$. The experimental shape-recovery process is illustrated in Fig.tre 2: the use of a thermocouple positioned very close to the sample allowed us to control the $T_{\text {iso }}$ in the oven. The process was recorded using a high resolution camera and analyszed frame by frame (one frame per second of the video). The opening angle was measured as shown in Fig.ure 1(b). The percentage of the original shape recovered $(\% S R)$ was obtained for each frame using equation (3), where $\varnothing$ is the opening angle, $\emptyset_{0}$ is the initial angle and $\Delta \emptyset_{\mathrm{t}}$ is the angle increase at a time $t_{\Delta} \Delta \emptyset_{\mathrm{t}}$ $\left.=\emptyset_{t}-\varnothing_{0}\right)$.

The shape-recovery time $\left(t_{s r}\right)$ was calculated as the time between the onset point $\left(t_{\text {onset }}\right)$ and the end point $\left(t_{\text {end }}\right)$ of the shape-recovery curve using equation $(4):-$

$$
(\% S R)_{t}=\frac{\Delta \emptyset_{t}}{180^{\circ}-\emptyset_{0}} \cdot 100
$$




$$
t_{\text {sr }}=t_{\text {end }}-t_{\text {onset }}
$$

a)

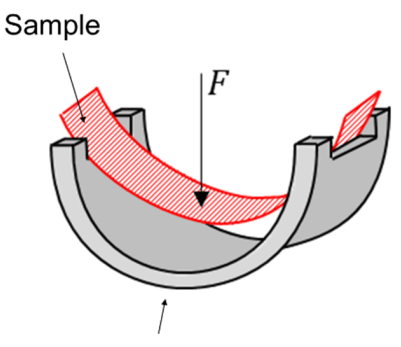

Bending device

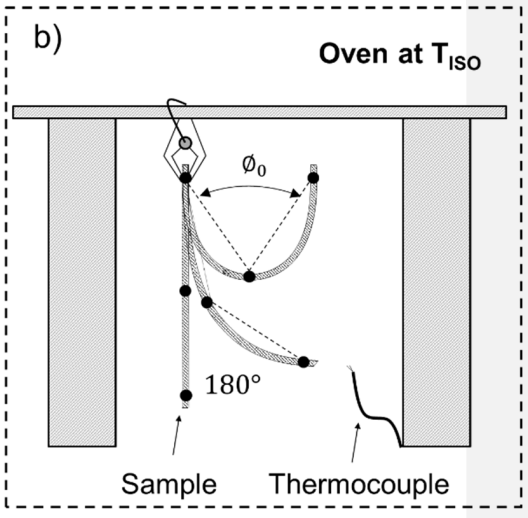

Figure 1. (a) Schematic representation of the bending device; (b) shape-recovery scenario
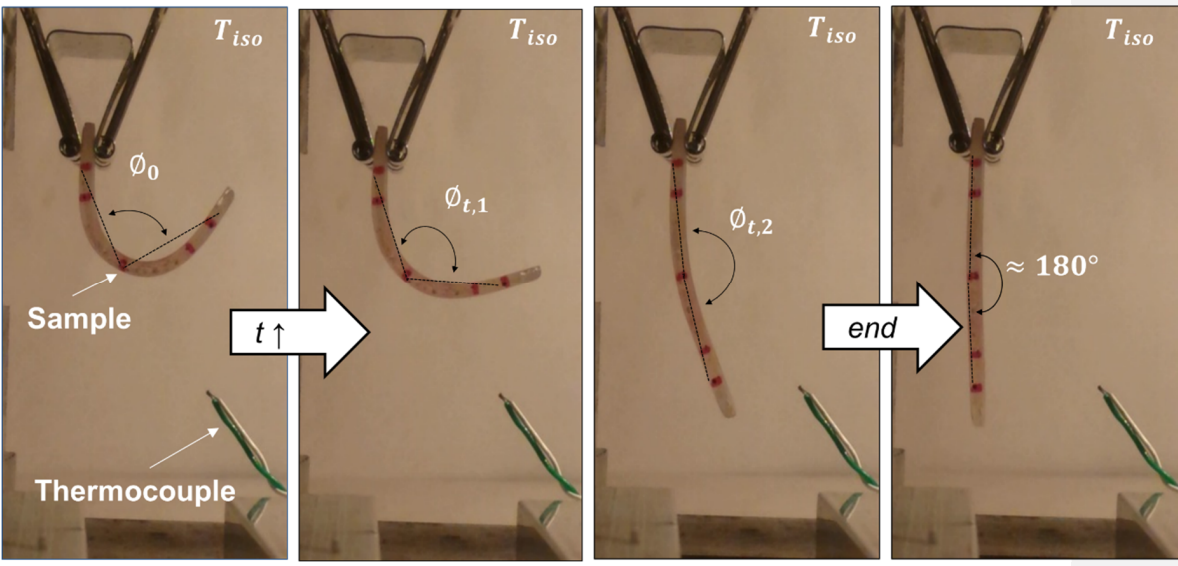

Figure 2. Images of the shape-recovery process at different shape-recovery times (from the beginning to the end)

\subsection{Determination of the sample temperature profile}

Overall, the isothermal-recovery experiments are limited by the sample temperature stabilization. Depending on the sample's dimensions and the heating conditions, the stabilization time can exceed the shape-recovery time, in which case the experiment becomes non-isothermal. To this end, in order to correctly analyse the shape-recovery process, the temperature on the surface of the sample was measured using a thermocouple stuck to the surface of one testing sample. 
The temperature was monitored from the initial time (placement of the sample inside the preheated oven) until the isothermal-recovery temperature was reached. Different $T_{\text {iso }}\left(50^{\circ} \mathrm{C}, 60^{\circ} \mathrm{C}\right.$ and $\left.70^{\circ} \mathrm{C}\right)$ were chosen to experimentally determine the coefficient of convection $(h)$ inside the oven and, therefore, to obtain the sample temperature profile. Assuming the sample is sufficiently thin and the heat resistance inside the sample sufficiently low $_{2}$ - that is, the temperature profile is uniform throughout the sample thickness $s_{2}-$ the energy balance shown in eq. (5) for the heating of the sample would hold true. Parameter $m$ is the mass of the sample, $C_{p}$ is the heat capacity of the material, $h$ is the coefficient of convection, $A$ is the area of the surface receiving the heat flow, $T$ is the sample surface temperature and $T_{\infty}$ is the isothermal temperature inside the oven.

$$
m \cdot C_{p} \cdot \frac{d T}{d t}=-h \cdot A \cdot\left(T-T_{\infty}\right)
$$

Integration of equation (5) with initial conditions of $T_{0}$ equal to the sample temperature at time 0 , before the sample is placed inside the oven, and boundary condition $T_{\infty}$ ias constant and equal to the temperature inside the preheated oven, leads to equation (6). Note that the experiments were performed inside an aircontrolled room, with $T_{0}=18^{\circ} \mathrm{C}$. Constant $" C$ " can be calculated from the slope of the plot $\ln \left(T-T_{\infty} / T_{0}-T_{\infty}\right)$ against time:-

$$
\ln \left(\frac{T-T_{\infty}}{T_{0}-T_{\infty}}\right)=-\frac{h \cdot A}{m \cdot C_{p}} \cdot t=-C \cdot t
$$

Nevertheless, the assumption of uniform temperature inside the sample should be validated using the Biot number $B i$ (see equation (7)), which should be less than 0.1 . After constant " $\mathrm{C}$ " has been determined, the convection coefficient $h$ and the Biot number can be estimated, using typical properties for epoxy systems (Pascault et al. 2002) (thermal conductivity of the sample $k=0.2 \mathrm{~W} / \mathrm{m} \cdot \mathrm{K}$, specific heat capacity $C_{p}=1300 \mathrm{~J} / \mathrm{kg} \cdot \mathrm{K}$ and density $\left.\rho=1200 \mathrm{kKg} / \mathrm{m}^{3}\right)$ and the sample dimensions, which are measured:-

$$
B i=\frac{h \cdot L}{k}
$$

Formatted: Font: Not Italic

Formatted: Font: Not Italic

Formatted: Font: Not Italic
Formatted: Font: Not Italic




\section{Results and discussion}

\subsection{Thermo-mechanical properties}

The results of the dynamic mechanical thermal analysis are shown in Fig.ure 3 , and the parameters of interest are summarized in Table 2. As discussed in our previous study (Belmonte et al. 2015), by using curing agents of higher functionality (4thiol instead of 3 thiol), the $T_{g}$ can be increased. Although the network strands have the same length, the higher functionality of the crosslinking points leads to a higher concentration of effective network strands in the system $v_{e}$, therefore increasing $T_{g}$. In addition, the incorporation of isocyanurate into the system also increases the $T_{g}$ because it acts as a crosslinking point by itself, increasing the crosslinking density and reducing the network strand length. As it was reported in the previous work (Belmonte et al. 2015), the use of iso in the 3thiol formulations led to some incompatibility issues, producing a broadening of the relaxation at $>30 \%$ of iso content. In order to avoid erroneous relationships between the shape-memory response and the network structure, we have chosen formulations with homogeneous network structures (as it can be deduced from the FHWM and $\tan \delta$ peak values in Table 2).

Table 2. Network structure and thermo-mechanical properties of the different materials of study

${ }^{\text {a }}$ Calculated using eq. (2)

${ }^{b}$ Calculated by integrating the tan $\delta$ curve into the temperature range corresponding to $0.1 \%$ and $2 \%$ of the completion (explained in the materials and methods section)

\begin{tabular}{|c|c|c|c|c|c|c|}
\hline Formulation & $\begin{array}{c}\mathbf{T}_{\mathrm{g}} \\
\left({ }^{\circ} \mathbf{C}\right)\end{array}$ & $\begin{array}{l}\mathbf{T}_{\mathrm{g}} \mathbf{E}^{\prime} \\
\left({ }^{\circ} \mathbf{C}\right)\end{array}$ & $\begin{array}{c}\text { FWHM } \\
\left({ }^{\circ} \mathrm{C}\right)\end{array}$ & $\begin{array}{l}\tan \delta \\
\text { peak }\end{array}$ & $\begin{array}{c}v_{\mathrm{e}^{\mathrm{a}}} \\
(\mathrm{mol} / \mathrm{kg})\end{array}$ & $\Delta T_{\text {net }}\left({ }^{o} \mathbf{C}\right)=\left(T_{0.1 \%}-T_{2 \%}\right)^{b}$ \\
\hline 3thiol-NEAT & 44.1 & 37.7 & 10.3 & 1.28 & 0.530 & $5.3=(31.6-36.9)$ \\
\hline 3thiol-(30\%)iso & 56.2 & 46.6 & 12.5 & 1.18 & 0.863 & $8.0=(35.6-43.6)$ \\
\hline 4thiol-NEAT & 59.7 & 52.0 & 12.0 & 1.06 & 0.822 & $8.7=(35.3-44.0)$ \\
\hline 4thiol-(30\%)iso & 75.4 & 68.2 & 11.8 & 1.05 & 1.209 & $16.5=(46.6-63.1)$ \\
\hline
\end{tabular}




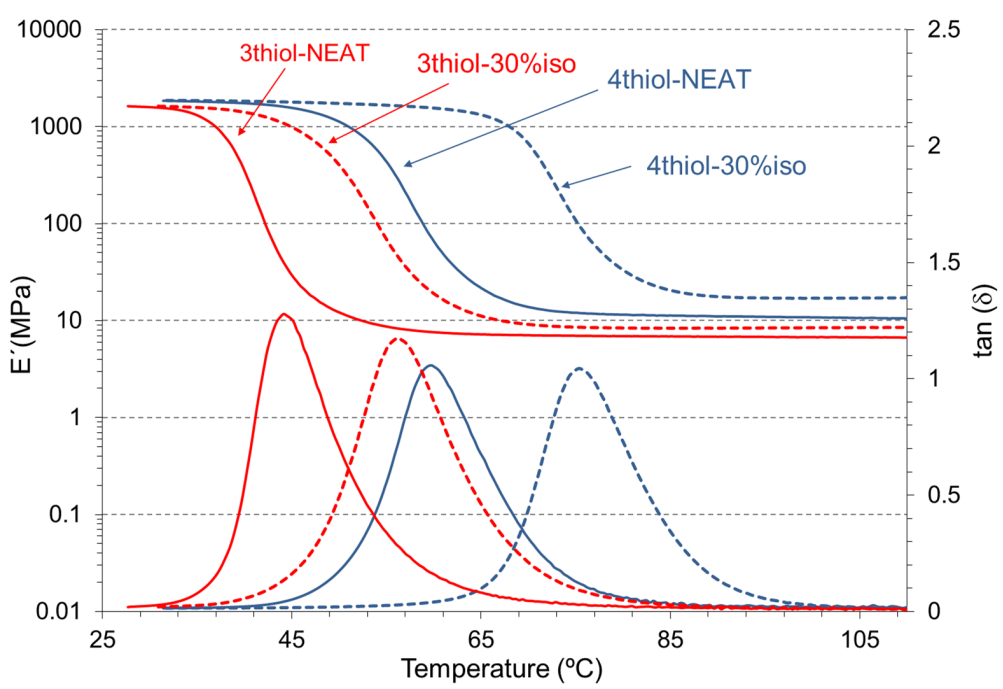

Figure 3. Dynamic mechanical thermal analyses of the different formulations of study

\subsection{Sample temperature profile}

Before discussing the results of the isothermal-recovery experiments, the temperature profile should be determined so that the shape-recovery process can be correctly analysed. Following the experimental procedure described in the materials and methods section, various $T_{i s o}$ were tested to calculate the constant $C$ in the oven and to get the temperature profile. In Fig.ure 4, the plot of $\ln \left(T-T_{\infty} / T_{0-}\right.$ $T_{\infty}$ ) against time is shown for different $T_{i s o}$. The data obtained show a very good correlation above $t=7 \mathrm{~s}$, with only slight deviations at the beginning. Since the shape-recovery processes (mostly those at higher $T_{i s o}$ ) took place rapidly after the sample had been placed in the oven, the temperature profile was divided into two different sections, equations (8) and (9), and the slope calculated separately for $\mathrm{t}<$ $7 \mathrm{~s}$ and $\mathrm{t} \geq 7 \mathrm{~s}$. The result was $C_{0}(t<7)=0.04 \mathrm{~s}^{-1}$ and $C(t \geq 7)=0.0243 \mathrm{~s}^{-1}$ :-

\begin{tabular}{l} 
Formatted: Not Highlight \\
Formatted: Not Highlight \\
\hline Formatted: Font: Not Italic
\end{tabular}

Formatted: Font: Not Italic

Formatted: Font: Not Italic

Formatted: Font: Not Italic

Formatted: Font: Not Italic 


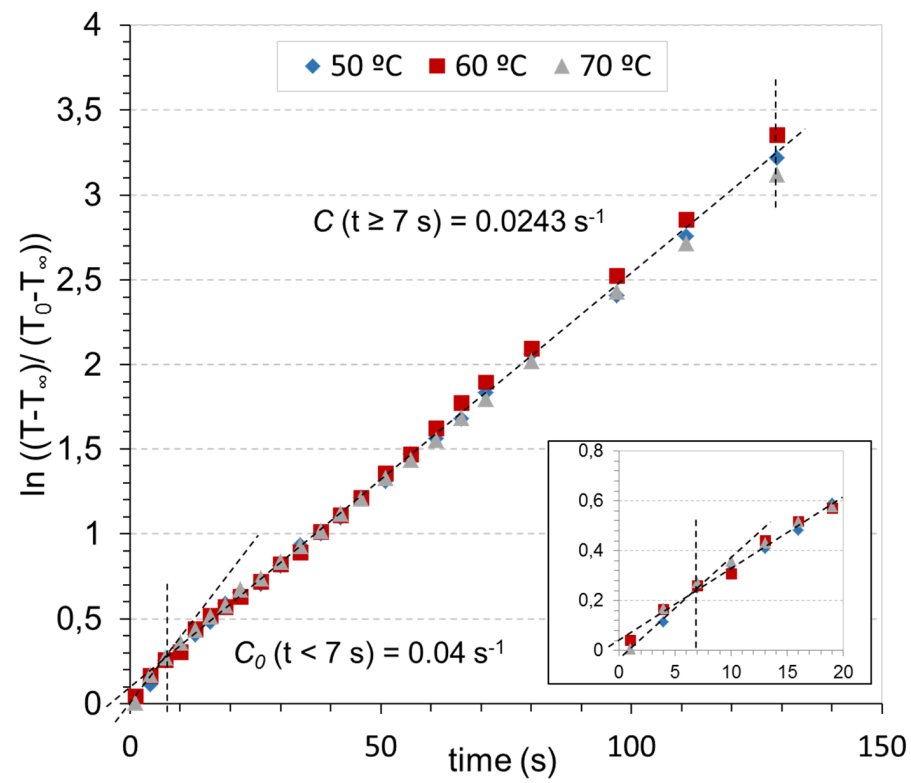

Figure 4. Curve fitting and determination of the constant $\mathrm{C}$ for each section of the temperature profile

$$
\begin{gathered}
\ln \left(\frac{T-T_{\infty}}{T_{0}-T_{\infty}}\right)=-\frac{h \cdot A}{m \cdot C_{p}} \cdot t=-C_{0} \cdot t \\
\ln \left(\frac{T-T_{\infty}}{T_{0}-T_{\infty}}\right)=-\frac{h \cdot A}{m \cdot C_{p}} \cdot t=-C \cdot(t-7) .
\end{gathered}
$$

Finally, in order to validate the assumption that temperature is uniform throughout the thickness of the sample, Bi should be less than 0.1. The coefficient of convection $h$ was calculated from constant " $C$ ”' by using values typical for epoxy systems (Pascault et al. 2002). The result was $h=28.4 \mathrm{~W} / \mathrm{m}^{2} \cdot \mathrm{K}$, so by applying the equation $(7), \mathrm{Bi} \approx 0.1$. Therefore, the error committed by assuming uniform temperature is minimal and fairly acceptable.

\subsection{Effect of shape-memory cycling on stability}

Once the temperature profile had been defined, experiments consisting of 10 consecutive cycles (programming the U-shape and recovering the original shape each time) were performed following the experimental setup shown in Figs.ure 1 and Figure 2 . In the programming process, two different $T_{\text {prog }}$ were chosen ${ }_{2} \div$ the $T_{g}{ }^{E}$ ' (shown to be the optimal mechanical point in our previous study (Belmonte et 
al. 2015) and other studies (Feldkamp and Rousseau 2010; Yakacki et al. 2008b)) and the $T_{g}$. For the shape-recovery process, $T_{i s o}$ was chosen to be equal to $T_{g}$. Because the same reference temperatures were used for each sample (in terms of the thermo-mechanical properties and network relaxation behaviour), the results were meaningful. The curves obtained are shown in Figsure. 5 and Figure 6, which also show the corresponding temperature profile. In addition, various parameters of interest are highlighted in the curves in Fig.ure 5(a) and summarized in Table 3: the temperature at which the shape-recovery process starts (stipulated as the onset point of the decrease in shape recovery) $\left(T_{\text {onset }}\right)$, the shaperecovery time $t_{s r}$, and the temperature range corresponding to the shape-recovery time $\left(\Delta T_{s r}\right)$ obtained using the sample temperature profile.

a)

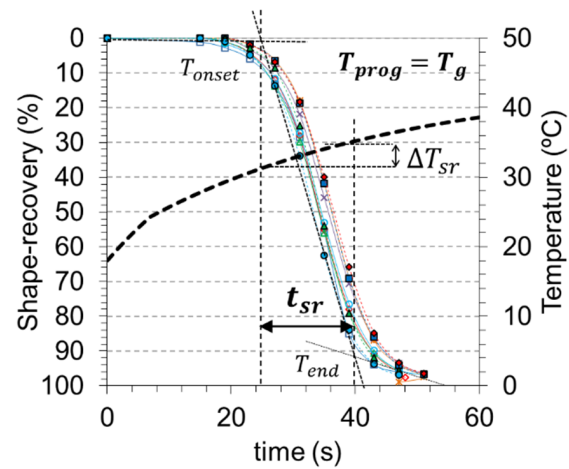

c)

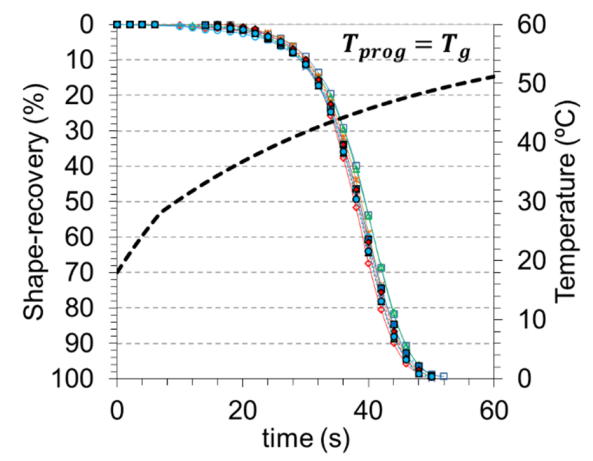

b)

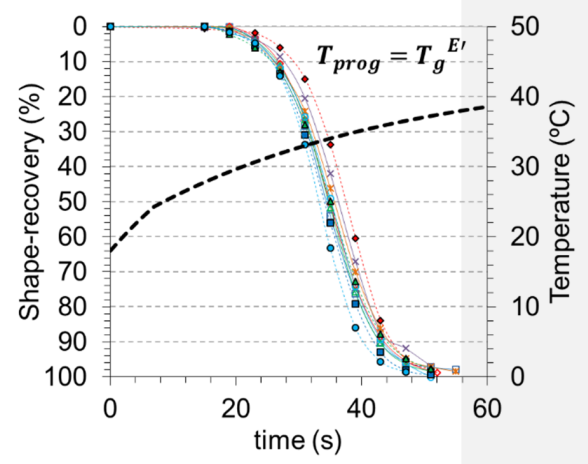

d)

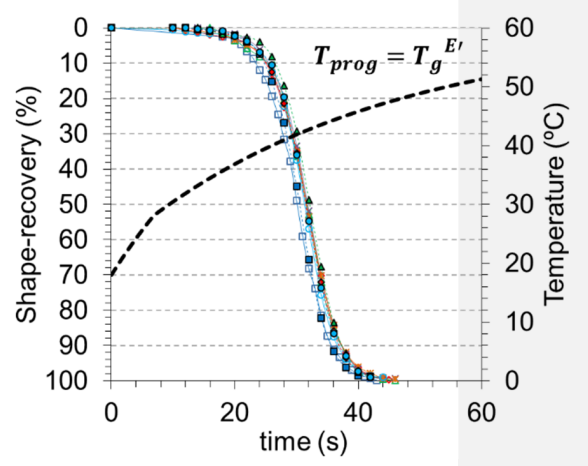

Figure 5. \% of shape recovery and the corresponding temperature profile (in discontinuous lines) as a function of the time over 10 consecutive cycles; $(\mathbf{a}, \mathbf{b}) 3$ thiol-NEAT programmed at $T_{\text {prog }}=T_{g}$ and $T_{g}{ }^{E^{\prime}} ; \mathbf{( c , d )} 4$ thiol-NEAT programmed at $T_{\text {prog }}=T_{g}$ and $T_{g}^{E^{\prime}}$. In all experiments $\mathrm{S}_{\mathrm{L}} T_{\text {iso }}=T_{g}$ 
a)

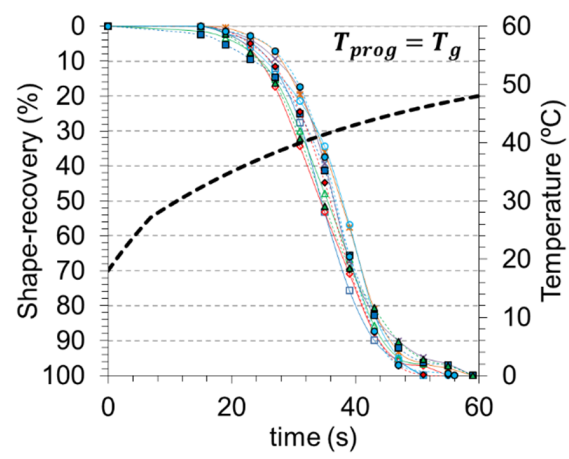

c)

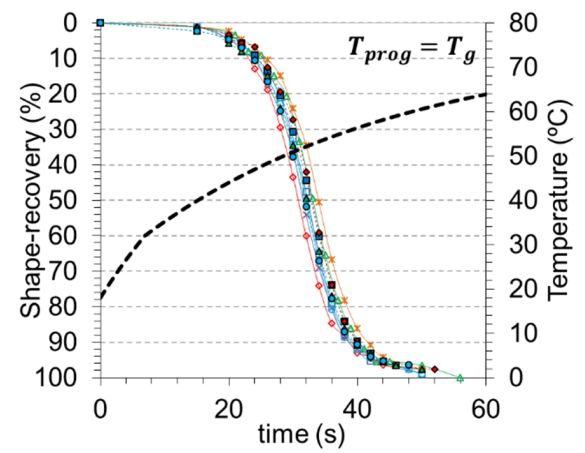

b)

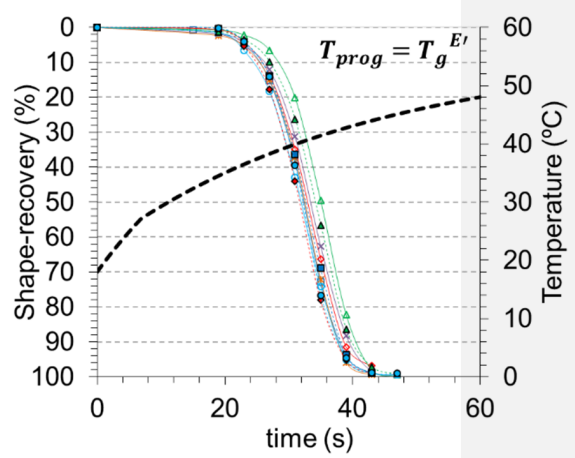

d)

4thiol-30\%iso

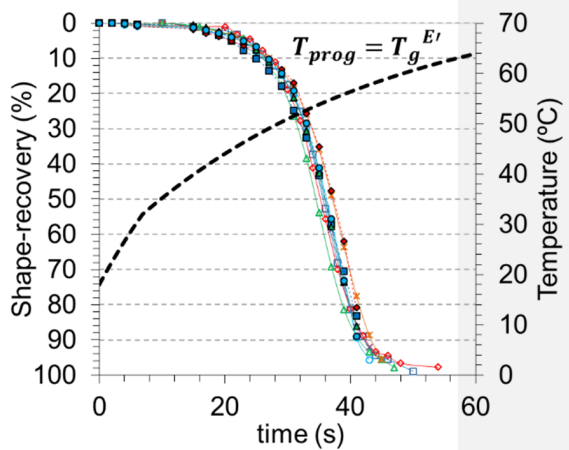

Figure $6 . \%$ of shape recovery and the corresponding temperature profile (in discontinuous lines) as a function of the time over 10 consecutive cycles; $(\mathbf{a}, \mathbf{b}) 3$ thiol-30\%iso programmed at $T_{\text {prog }}=T_{g}$ and $T_{g}^{E^{\prime}} ;$ (c,d) 4thiol-30\%iso programmed at $T_{\text {prog }}=T_{g}$ and $T_{g}^{E^{\prime}}$. In all experiments ${ }_{L} T_{\text {iso }}=T_{g}$

Table 3. Mean value and its deviation of the shape-recovery parameters for all the formulations studied

\begin{tabular}{c|c|c|c|c|c|c}
\cline { 2 - 7 } \multicolumn{2}{c|}{$\mathbf{T}_{\text {onset }}\left({ }^{\mathbf{o}} \mathbf{C}\right)$} & \multicolumn{2}{c|}{$\Delta \mathbf{T}_{\text {sr }}\left({ }^{\mathbf{}} \mathbf{C}\right)$} & \multicolumn{2}{c}{$\mathbf{t}_{\text {sr }}(\mathbf{s})$} \\
\hline Formulation & $\begin{array}{c}\mathbf{T}_{\text {prog }}= \\
\mathbf{T}_{\mathbf{g}}\end{array}$ & $\begin{array}{c}\mathbf{T}_{\text {prog }} \\
=\mathbf{T}_{\mathbf{g}} \mathbf{E}^{\prime}\end{array}$ & $\begin{array}{c}\mathbf{T}_{\text {prog }} \\
=\mathbf{T}_{\mathbf{g}}\end{array}$ & $\begin{array}{c}\mathbf{T}_{\text {prog }} \\
=\mathbf{T}_{\mathbf{g}} \mathbf{E}^{\prime}\end{array}$ & $\mathbf{T}_{\text {prog }}=\mathbf{T}_{\mathbf{g}}$ & $\mathbf{T}_{\text {prog }}=\mathbf{T}_{\mathbf{g}} \mathbf{E}^{\prime}$ \\
\hline 3thiol-NEAT & $32 \pm 0.9$ & $32 \pm 1.1$ & $3.0 \pm 0.2$ & $3.4 \pm 0.2$ & $12.0 \pm 0.8$ & $14.0 \pm 0.9$ \\
3thiol-30\%iso & $38 \pm 1.0$ & $38 \pm 1.0$ & $5.9 \pm 0.3$ & $5.6 \pm 0.1$ & $19.0 \pm 1.1$ & $15.0 \pm 0.4$ \\
\hline 4thiol-NEAT & $42 \pm 0.4$ & $40 \pm 0.4$ & $5.0 \pm 0.1$ & $4.6 \pm 0.1$ & $14.3 \pm 0.4$ & $11.0 \pm 0.4$ \\
4thiol-30\%iso & $48 \pm 0.7$ & $50 \pm 0.7$ & $6.1 \pm 0.1$ & $6.7 \pm 0.1$ & $11.0 \pm 0.3$ & $13.0 \pm 0.4$ \\
\hline
\end{tabular}

Figures 5 and Figure 6 clearly show that the shape-recovery process starts before the temperature of the sample stabilizes, so it is not isothermal. The temperature 
of the sample takes at least 2 minutes to stabilize, when the shape-recovery has already finished. This drawback is common when testing at isothermal-recovery temperatures near or above $T_{g}$. Pandini et al. (Pandini et al. 2013) observed the same phenomenon with epoxy-amine based SMPs, and stated that only the samples tested at $T_{i s o}<\left(T_{g}-15^{\circ} \mathrm{C}\right)$ proceed isothermally. Nevertheless, very few studies have taken into account the real temperature of the sample when analysing isothermal-recovery experiments. The temperature profile can be used to analyse the different stages during the shape-recovery process and determine the real temperature of the sample at which the shape-recovery process starts $T_{\text {onset }}$ (see Fig.mre 5(a)). As can be seen in Table 3, $T_{\text {onset }}$ increases as $T_{g}$ increases (see Table 2 from 3thiol-NEAT to 4 thiol-30\%iso), and does not vary with $T_{p r o g}\left( \pm 2^{\circ} \mathrm{C}\right.$ with no defined trend among the different formulations). It can also be seen that $T_{\text {onset }}$ is within the temperature range of the onset of network relaxation $\Delta T_{\text {net }}$ (see Table 2 ), thus suggesting that this onset triggers the shape-recovery process. Shaperecovery takes place in the temperature interval $\Delta T_{s r}$, and it can be seen that it is completed below $T_{g}\left(T_{\text {onset }}+\Delta T_{s r}<T_{g}\right)$. It must be remembered that the nominal value of $T_{g}$ determined in this study corresponds to the tan $\delta$ peak temperature, which is frequency dependent. The network relaxation process obviously starts before this temperature is reached. In overall terms, the shape-recovery curves can be divided into two different stages. At the beginning, when the sample is placed in the oven, the process is very slow because the sample temperature is well below the $T_{g}$ during this initial period. Once the sample has reached $T_{\text {onset }}$ and network relaxation starts to take place, the curves show an exponential decay of relaxation processes (Diani et al. 2012; Ge et al. 2012), further enhanced by the progressive temperature increase.

All the samples fully recovered their original shape through the cycling procedure and there was no loss of temporary shape after each cycle. The shape-fixation and shape-recovery performances were excellent regardless of $T_{\text {prog }}$, and stable over the 10 consecutive cycles. It should be pointed out that no differences were appreciated between the first cycle and the second cycle, thus suggesting that the network structure created after the curing process is stable from a mechanical and energy point of view, that no training procedure (Rousseau 2008) (thermomechanical accommodation) was necessary, and that no plastic and irreversible rearrangements within the network occur after the first cycle. On the other hand, 
focusing on the shape-recovery process, the $t_{s r}$ ranges from 11 to 19 seconds and shows great stability over the 10 consecutive cycles $( \pm 1.1 \mathrm{~S}$ - in the worst case) irrespective of $T_{\text {prog. }}$. The differences between programming at $T_{g}$ or $T_{g}{ }^{E^{\prime}}$ do not follow any specific trend ${ }_{2}$ and no cracking of the sample was observed. This high thermo-mechanical stability is provided by the excellent stability of the epoxybased materials (Liu et al. 2010). As can be seen in Fig.ure 5, the differences caused by the curing agent are almost negligible, so crosslinking points with different functionalities have little impact on stability. Likewise, introducing isocyanurate into the system (Fig.ture 6) is also negligible in terms of stability. These results reveal the good shape-memory response of these materials, which adds to the advantages of developing SMPs by thiol-epoxy "click" chemistry. Taking into account these results and the excellent mechanical properties of the materials (high ultimate stress and strain observed in our previous study (Belmonte et al. 2015)), these materials seem to be excellent candidates for actuator devices.

\subsection{Effect of the isothermal-recovery temperature on the shape- recovery process}

The effect of the isothermal-recovery temperature $T_{i s o}$ on the shape-recovery process was analysed using the same experimental setup as in the previous section. Various $T_{i s o}$, ranging from $\left(T_{g}-10\right.$ to $\left.T_{g}+20\right)$, were chosen to further analyse the effect of the network relaxation dynamics on the shape-recovery process. For all the experiments, given the results obtained in the previous section, $T_{\text {prog }}$ was chosen as the optimal mechanical point- $\left(T_{g}{ }^{\prime}\right)$. All the experiments were performed three times ${ }_{2}$ and the average value is presented. The curves obtained are shown in Fig.ure 7. Taking into account that the experiments proceed nonisothermally, besides the parameters in the previous section, the times for the

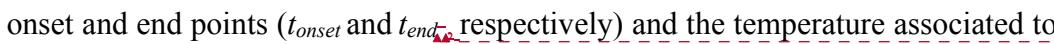
the end point $\left(T_{\text {end }}\right)$ were determined to properly analyse each stage of the process. All these parameters are summarized in Table 4 or represented in Fig.ure 8 with respect to $T_{\text {iso. }}$.
Formatted: Font: Not Italic

Formatted: Font: Not Italic

Formatted: Font: Not Italic

Formatted: Not Superscript/ Subscript

Formatted: Font: Not Italic, Not Superscript/ Subscript

Formatted: English (United Kingdom) 


$$
-\diamond-T_{g}-10-\square-T_{g}-5-\Delta-T_{g}-\bullet-T_{g}+5-\square-T_{g}+10-\square-T_{g}+15-\Delta-T_{g}+20
$$

a)

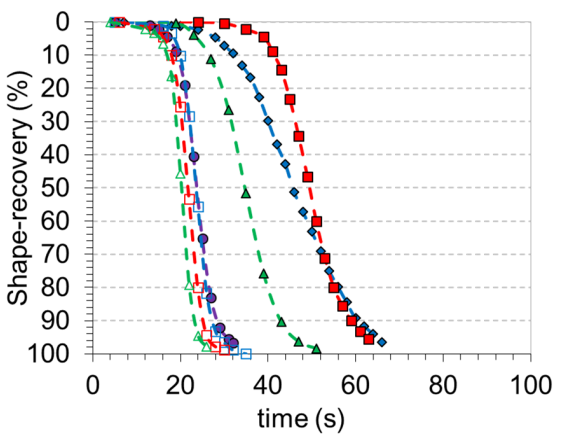

c)

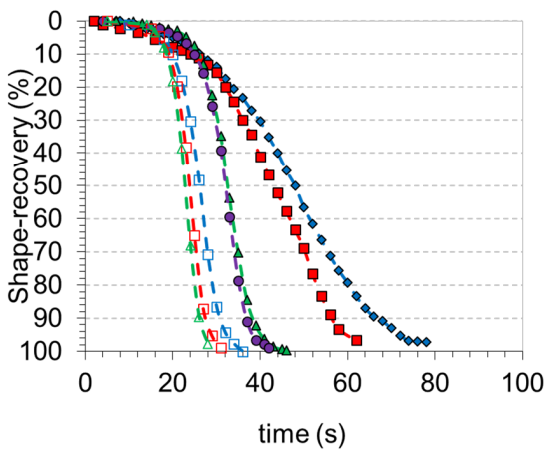

b) 3thiol-30\%iso

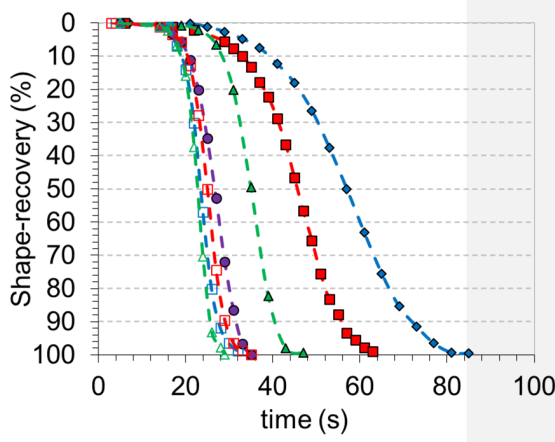

d)

4thiol-30\%iso

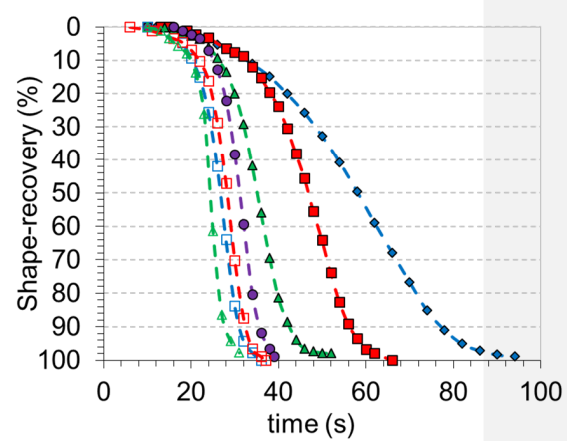

Figure 7. Shape-recovery curves for all the formulations at different $T_{\text {iso }}$ (from $T_{g}-10$ to $T_{g}+20$ ). All the samples were programmed at $T_{g}{ }^{E^{\prime}}$ 
a)

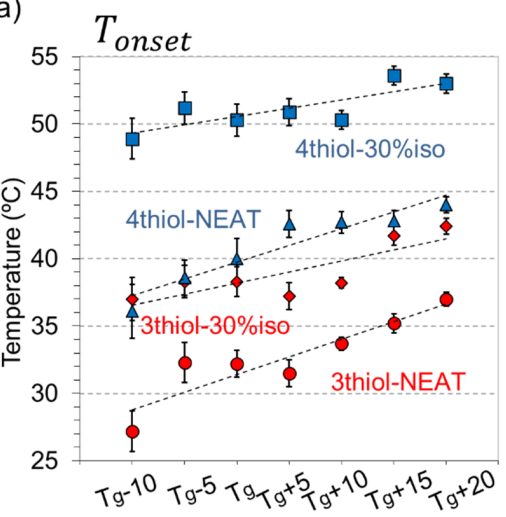

c)

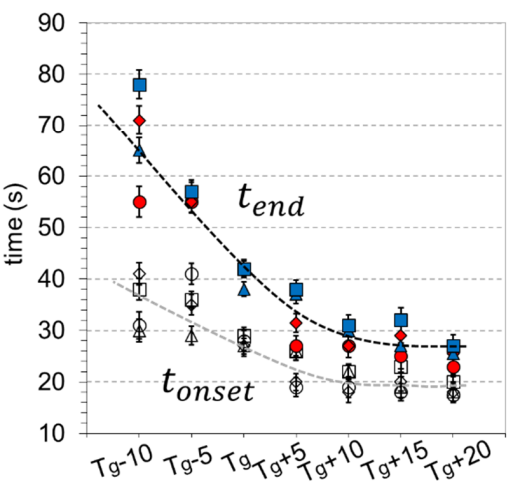

b)

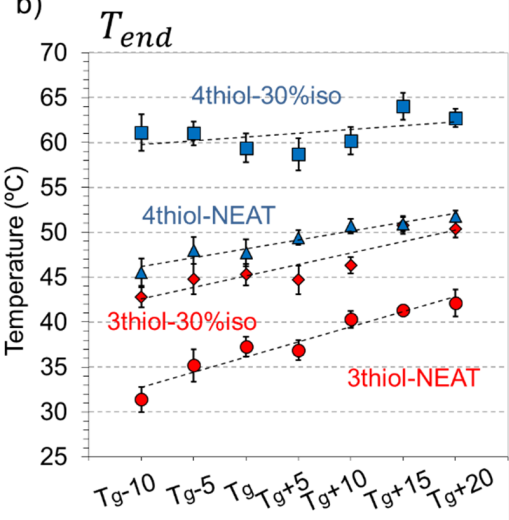

d)

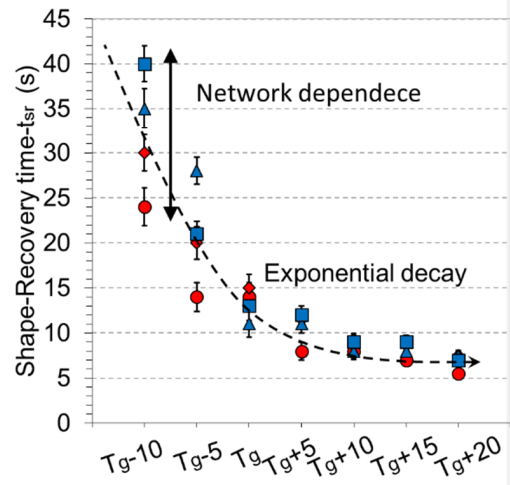

Figure 8. (a) Temperature corresponding to the onset point; (b) temperature corresponding to the end point; (c) times corresponding to the onset and end points; (d) shape-recovery time $\left(t_{s r}\right)$. All data is shown including the standard deviation.

Table 4. Average value of the parameters of interest determined at different $T_{\text {iso }}$ for all the formulations of study: the time corresponding to the onset point, $t_{\text {onset, }}$ the temperature range for the shape-recovery, $\Delta \mathrm{T}_{\mathrm{sr}}$, and the shape-recovery time, $\mathrm{t}_{\mathrm{sr}}$. Maximum standard deviation of \pm 2-o $\mathrm{C}$ in $\mathrm{T}_{\text {onset }}$ and $\mathrm{T}_{\text {end, }} \pm 3$ seconds in $\mathrm{t}_{\mathrm{sr}} \pm 0$, $8-\mathrm{-} \mathrm{C}$ in $\Delta \mathrm{T}_{\mathrm{sr}}$ and \pm 3 seconds in $t_{\text {onset }}$.

\begin{tabular}{|c|c|c|c|c|c|c|c|c|c|c|c|c|}
\hline & \multicolumn{3}{|c|}{ 3thiol-NEAT } & \multicolumn{3}{|c|}{ 3thiol-30\%iso } & \multicolumn{3}{|c|}{ 4thiol-NEAT } & \multicolumn{3}{|c|}{ 4thiol-30\%iso } \\
\hline & $\begin{array}{l}\boldsymbol{t}_{\text {onset }} \\
\text { (s) }\end{array}$ & $\begin{array}{l}\Delta \boldsymbol{T}_{\boldsymbol{s} r} \\
\left({ }^{\circ} \mathrm{C}\right)\end{array}$ & $\begin{array}{c}\boldsymbol{t}_{\boldsymbol{s} r} \\
(\mathrm{~s})\end{array}$ & $\begin{array}{c}\boldsymbol{t}_{\text {onset }} \\
\text { (s) }\end{array}$ & $\begin{array}{l}\Delta \boldsymbol{T}_{\boldsymbol{s}} \\
\left({ }^{\circ} \mathrm{C}\right)\end{array}$ & $\begin{array}{l}\boldsymbol{t}_{\boldsymbol{s} \boldsymbol{r}} \\
(\mathrm{s})\end{array}$ & $\begin{array}{c}\boldsymbol{t}_{\text {onset }} \\
\text { (s) }\end{array}$ & $\begin{array}{l}\Delta \boldsymbol{T}_{\boldsymbol{s} r} \\
\left({ }^{\circ} \mathrm{C}\right)\end{array}$ & $\begin{array}{l}\boldsymbol{t}_{\boldsymbol{s} r} \\
(\mathrm{~s})\end{array}$ & $\begin{array}{c}\boldsymbol{t}_{\text {onset }} \\
\text { (s) }\end{array}$ & $\begin{array}{l}\Delta \boldsymbol{T}_{\boldsymbol{s} r} \\
\left({ }^{\circ} \mathrm{C}\right)\end{array}$ & $\begin{array}{l}\boldsymbol{t}_{\boldsymbol{s} r} \\
(\mathrm{~s})\end{array}$ \\
\hline Tg-10 & 31 & 4.2 & 24.0 & 41 & 5.8 & 30.0 & 30 & 9.4 & 35.0 & 38 & 12.2 & 40.0 \\
\hline Tg-5 & 41 & 2.9 & 14.0 & 35 & 6.5 & 20.0 & 29 & 9.4 & 28.0 & 36 & 9.8 & 21.0 \\
\hline $\mathrm{Tg}$ & 28 & 5.1 & 14.0 & 27 & 7.0 & 15.0 & 27 & 7.7 & 11.0 & 29 & 9.1 & 13.0 \\
\hline $\mathrm{Tg}+5$ & 19 & 5.4 & 8.0 & 20 & 7.5 & 11.5 & 26 & 6.8 & 11.0 & 26 & 7.8 & 12.0 \\
\hline $\mathrm{Tg}+10$ & 19 & 6.6 & 8.0 & 18 & 8.1 & 9.0 & 22 & 8.0 & 8.0 & 22 & 9.9 & 9.0 \\
\hline $\mathrm{Tg}+15$ & 18 & 6.1 & 7.0 & 20 & 9.1 & 9.0 & 19 & 8.1 & 8.0 & 23 & 10.4 & 9.0 \\
\hline$T g+20$ & 17 & 5.1 & 5.5 & 18 & 8.0 & 7.5 & 18 & 7.8 & 7.5 & 20 & 9.7 & 7.0 \\
\hline
\end{tabular}


In general, regardless of $T_{i s o}$, all the curves go through two different stages as discussed above: the initial, slow stage until $T_{\text {onset }}$ is reached followed by the typical exponential decay of the relaxation processes. The time for this initial stage $t_{\text {onset }}$ decreases as $T_{\text {iso }}$ increases (see Table $4, t_{\text {onset }}$ decreases by $23 \mathrm{~s}$ in the case of 3thiol-30\%iso from $T_{g-10}$ to $T_{g}+20$ ) because the temperature increases more quickly when the sample is placed in the oven preheated to a higher temperature. As a consequence of this quick increase in the sample temperature in the early stage, $T_{\text {onset }}$ is also slightly displaced to higher temperatures as $T_{\text {iso }}$ increases in a linear fashion (see Fig. 3thiol-NEAT formulation, from $26^{\circ} \mathrm{C}$ to $37^{\circ} \mathrm{C}$ ). Nevertheless, it should be pointed out that in all cases, these temperatures are within the temperature range given by $\Delta T_{n e t}$, so the shape-recovery process takes place because the network relaxation is activated. The shape-recovery time, $t_{s r}$, naturally decreases due to the enhancement of the network relaxation dynamics as $T_{i s o}$ increases (Williams et al. 1955), which leads to a faster temperature increase in the sample taking place below and during the glass transition process. This phenomenon can be observed in Fig.ure 8(d), where $t_{s r}$ decreases as $T_{i s o}$ increases and decays exponentially (as expected from the shape-recovery curves). In contrast, as can be seen in Table 4, the temperature range in which recovery takes place, $\Delta T_{s r}$, does not show any specific trend. As is observed in Fig.tre 8(b), the trend in the temperature $T_{\text {end }}$ (which corresponds to $T_{\text {onset }}+\Delta T_{s r}$ ) is very similar to that of $T_{\text {onset }}$ (Fig.ure 8(a)): it slightly increases as $T_{i s o}$ increases, but is still below $T_{g}$. It should be pointed out that all the materials complete the shape-recovery process at $T_{i s o}<T_{g}$. However, it must be remembered that the nominal value of $T_{g}$ corresponding to the $\tan _{-} \delta$ peak temperature is frequency_-dependent and is only taken as a reference: it does not mean that network relaxation cannot take place below this temperature; $e_{2}$ it is just a question of time. To sum up, an increase in $T_{\text {iso }}$ produces a faster temperature increase in the samples, leading to faster network relaxation and recovery and a shift in the shape-recovery curve to higher temperatures. However, this takes place in narrow temperature ranges.

Differences in the shape-recovery time in the various formulations (see Fig.ure 8 (d)) are negligible at $T_{i s o} \gg T_{g}$ because the fast temperature increase taking place before and during the glass transition process leads to very fast relaxation dynamics in all cases. In contrast, at $T_{i s o}<T_{g_{2}}$ the fast temperature increase

Formatted: Font: Not Italic
Formatted: Font: Not Italic

Formatted: Not Superscript/ Subscript

Formatted: Not Highlight

Formatted: Not Highlight

Formatted: Font: Not Italic, Not Superscript/ Subscript 
(mostly taking place in the formulations with the highest $\mathrm{T}_{\mathrm{g}}$ ) happens well-below the glass transition and therefore has a minor role, whereas the differences due to the network structure are considerable. The formulations with 3thiol show lower values of $t_{s r}$ than those with 4 thiol. This may be caused by the higher functionality of the 4thiol which leads to a more hindered spatial structure and more difficult relaxation, while the 3thiol crosslinker leads to a less packed structure with higher free volume, thus, accelerating the network relaxation process (Pandini et al. 2013). As a result, the $t_{s r}$ differences increase to $16 \mathrm{~s}$ from 3thiol-NEAT to 4thiol-30\%iso formulations. In terms of tuning the shape-recovery process, differences in shape-recovery time between the formulations are maximized by working at $T_{\text {iso }}<T_{g}$. Recovery is fast in all cases at $T_{i s o}>T_{g}$ for all the formulations, so the difference comes from the different network structure which leads to different transition temperatures. The materials studied here are promising not only because they ensure that shape recovery can be completed at low temperatures (i.e. if a slow ending is desired), but also because of their tuneable network structure properties and shape-recovery behaviour.

\section{Conclusions}

Shape-memory polymers based on "thiol-epoxy" networks with tailorable glass transition and crosslinking density have been developed by "click" chemistry and using latent initiators (encapsulated imidazoles). These materials have homogeneous network structures and narrow relaxation processes, a significant

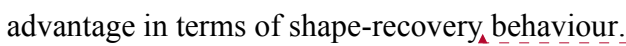

Formatted: English (United Kingdom)

The results of the shape-memory cycling of the samples revealed a highly stable thermo-mechanical and shape-recovery time for all the materials regardless of the programming temperature $\left(T_{g}^{E^{\prime}}\right.$ or $\left.T_{g}\right)$, which suggests that the mechanical response can be optimized (by programming at $T_{g}{ }^{E}$ ) while high stability can be maintained for applications involving repetitive use of an actuator.

Shape recovery could not be activated under strictly isothermal conditions because the time the sample took to reach the isothermal recovery temperature $T_{\text {iso }}$ inside the oven was much longer than the time taken to complete the shape recovery. The shape-recovery process takes place in two well-defined stages: initially, when the sample temperature is still low, the temporary shape remains 
stable $_{2}$ but when the sample temperature increases and network relaxation is activated, shape recovery takes place very fast and follows the typical exponential decay of the relaxation processes until completion. The shape-recovery process is accelerated but shifted to slightly higher temperatures when the isothermalrecovery temperature $T_{i s o}$ was increased due to the faster and higher increase in the sample temperature, leading to faster relaxation dynamics. At isothermalrecovery temperatures $T_{i s o}$ well above the $T_{g}$ of the samples, the effect of the temperature on the shape-recovery time was negligible. The effect of the different network structures of the materials was found to be greater when recovering at $T_{i s o}$ $<T_{g}$. Formulations with higher crosslinking densities and more hindered network structures have slower shape-recovery rates due to slower relaxation dynamics at low temperatures. These results suggest that the shape-recovery process can be controlled by changing the network structure and working at $T_{i s o}<T_{g}$ to maximize the effect of the structure and/or by increasing $T_{i s o}$ to minimize the effect but increase the shape-recovery rate.

Acknowledgements The authors would like to thank MICINN (MAT2014-53706-C03-01 and MAT2014-53706-C03-02) and Generalitat de Catalunya (2014-SGR-67) for financial support.

\section{References}

Arrieta, J.S., Diani, J., Gilormini, P.: Cyclic and monotonic testing of free and constrained recovery properties of a chemically crosslinked acrylate. J. Appl. Polym. Sci. 131, 39813_-39820 (2014)(a).

Arrieta, S., Diani, J., Gilormini, P.: Experimental characterization and thermoviscoelastic modeling of strain and stress recoveries of an amorphous polymer network. Mech. Mater. 68, 95-103 (2014)(b).

Belmonte, A., Guzmán, D., Fernández-Francos, X., De la Flor, S.: Effect of the Network Structure and Programming Temperature on the Shape-Memory Response of Thiol-Epoxy "Click" Systems. Polymers. 2146-2164 (2015).

Berg, G.J., McBride, M.K., Wang, C., Bowman, C.N.: New directions in the chemistry of shape memory polymers. Polymer. 55, 1-24 (2014).

Binder, W.H., Sachsenhofer, R.: "Click" chemistry in polymer and materials science. Macromol. Rapid Commun. 28, 15-54 (2007).

Brändle, A., Khan, A.: Thiol-epoxy "click" polymerization: efficient construction of reactive and functional polymers. Polym. Chem. 3, 3224 (2012).

Carlborg, C.F., Vastesson, A., Liu, Y., Van Der Wijngaart, W., Johansson, M., Haraldsson, T.: Functional off-stoichiometry thiol-ene-epoxy thermosets featuring temporally controlled curing stages via an UV/UV dual cure process. J. Polym. Sci. Part A Polym. Chem. 52, 2604-2615 (2014).

Chen, X., Nguyen, T.D.: Influence of thermoviscoelastic properties and loading conditions on the recovery performance of shape memory polymers. Mech. Mater. 43, 127-138 (2011).

Diani, J., Gilormini, P., Frédy, C., Rousseau, I.: Predicting thermal shape memory of crosslinked polymer networks from linear viscoelasticity. Int. J. Solids Struct. 49, 793-799 (2012). 
014804 (2015).

Fan, M., Yu, H., Li, X., Cheng, J., Zhang, J.: Thermomechanical and shape-memory properties of epoxy-based shape-memory polymer using diglycidyl ether of ethoxylated bisphenol-A. Smart Mater. Struct. 22, 055034 (2013).

Feldkamp, D.M., Rousseau, I. A.: Effect of the deformation temperature on the shape-memory behavior of epoxy networks. Macromol. Mater. Eng. 295, 726-734 (2010).

Feldkamp, D.M., Rousseau, I. A.: Effect of Chemical Composition on the Deformability of ShapeMemory Epoxies. Macromol. Mater. Eng. 296, 1128-1141 (2011).

Flores, M., Tomuta, A.M., Fernández-Francos, X., Ramis, X., Sangermano, M., Serra, A.: A new two-stage curing system: Thiol-ene/epoxy homopolymerization using an allyl terminated hyperbranched polyester as reactive modifier. Polymer. 54, 5473-5481 (2013).

Ge, Q., Yu, K., Ding, Y., Jerry Qi, H.: Prediction of temperature-dependent free recovery behaviors of amorphous shape memory polymers. Soft Matter. 8, 11098 (2012).

Guzmán, D., Ramis, X., Fernández-Francos, X., Serra, A.: New Catalysts For Diglycidyl Ether Of Bisphenol A Curing Based On Thiol-Epoxy Click Reaction. Eur. Polym. J. (2014).

Hager, M.D., Bode, S., Weber, C., Schubert, U.S.: Shape memory polymers: Past, present and future developments. Prog. Polym. Sci. 49--50, 3-33 (2015).

Jin, K., Heath, W.H., Torkelson, J.M.: Kinetics of multifunctional thiol-epoxy click reactions studied by differential scanning calorimetry: Effects of catalysis and functionality. Polymer. 81, 70-78 (2015).

Lakhera, N., Yakacki, C.M., Nguyen, T.D., Frick, C.P.: Partially constrained recovery of (meth)acrylate shape-memory polymer networks. J. Appl. Polym. Sci. 126, 72-82 (2012).

Lendlein, A., Sauter, T.: Shape-memory effect in polymers. Macromol. Chem. Phys. 214, 11751177 (2013).

Leng, J., Yu, K., Sun, J., Liu, Y.: Deployable morphing structure based on shape memory polymer. Aircr. Eng. Aerosp. Technol. 87, 218-223 (2015).

Leonardi, A.B., Fasce, L. A., Zucchi, I. A., Hoppe, C.E., Soulé, E.R., Pérez, C.J., Williams, R.J.J.: Shape memory epoxies based on networks with chemical and physical crosslinks. Eur. Polym. J. 47, 362-369 (2011).

Liu, Y., Han, C., Tan, H., Du, X.: Thermal, mechanical and shape memory properties of shape memory epoxy resin. Mater. Sci. Eng. A. 527, 2510-2514 (2010).

Loureiro, R.M., Amarelo, T.C., Abuin, S.P., Soulé, E.R., Williams, R.J.J.: Kinetics of the epoxy-thiol click reaction initiated by a tertiary amine: Calorimetric study using monofunctional components. Thermochim. Acta. 616, 79-86 (2015).

Pandini, S., Bignotti, F., Baldi, F., Passera, S.: Network architecture and shape memory behavior of cold-worked epoxies. J. Intell. Mater. Syst. Struct. 24, 1583-1597 (2013).

Pascault, J.-P., Sautereau, H., J, V., R, W.J.J.: Thermosetting Polymers. CRC Press (2002).

Rousseau, I. A., Xie, T.: Relationship between materials properties and shape memory behavior in epoxy-amine polymers. In: Materials Research Society Symposium Proceedings. pp. 31-36 (2009).

Rousseau, I.A.: Challenges of shape memory polymers: A review of the progress toward overcoming SMP's limitations. Polym. Eng. Sci. 48, 2075-2089 (2008).

Santhosh Kumar, K.S., Biju, R., Reghunadhan Nair, C.P.: Progress in shape memory epoxy resins. React. Funct. Polym. 73, 421-430 (2013).

Santiago, D., Fabregat-Sanjuan, A., Ferrando, F., De la Flor, S.: Recovery stress and work output 
in hyperbranched poly(ethyleneimine)-modified shape-memory epoxy polymers. J. Polym. Sci. Part B Polym. Phys. 54(10), 1002-1013 (2016).

Santiago, D., Fernández-Francos, X., Ferrando, F., De la Flor, S.: Shape-memory effect in hyperbranched poly(ethyleneimine)-modified epoxy thermosets. J. Polym. Sci. Part B Polym. Phys. 924-933 (2015).

Scalet, G., Auricchio, F., Bonetti, E., Castellani, L., Ferri, D., Pachera, M., Scavello, F.: An experimental, theoretical and numerical investigation of shape memory polymers. Int. J. Plast. 67, 127-147 (2015).

Song, J.J., Chang, H.H., Naguib, H.E.: Biocompatible shape memory polymer actuators with high force capabilities. Eur. Polym. J. 67, 186-198 (2015).

Sun, L., Huang, W.M., Ding, Z., Zhao, Y., Wang, C.C., Purnawali, H., Tang, C.: Stimulus-responsive shape memory materials: A review. Mater. Des. 33, 577-640 (2012).

Tandon, G.P., Gibson, T., Shumaker, J., Coomer, R., Baur, J., Justice, R.S.: Processing and characterization of novel bismaleimide-based shape memory polymer composites. ASME 2012 Conference on Smart Materials, Adaptive Structures and Intelligent Systems, SMASIS 2012. 1,19_ -25 (2012).

Tandon, G.P., McClung, A.J., Bauer, J.W.: Shape-Memory Polymers for Aerospace Applications. DEStech Publications (2016).

Williams, M.L., Landel, R.F., Ferry, J.D.: The Temperature Dependence of Relaxation Mechanisms in Amorphous Polymers and Other Glass-forming Liquids. J. Am. Chem. Soc. 77, 3701-3707 (1955)

Yakacki, C.M., Shandas, R., Lanning, C., Rech, B., Eckstein, A., Gall, K.: Unconstrained recovery characterization of shape-memory polymer networks for cardiovascular applications. Biomaterials. 28, 2255-2263 (2007).

Yakacki, C.M., Shandas, R., Safranski, D., Ortega, A.M., Sassaman, K., Gall, K.: Strong, tailored, biocompatible shape-memory polymer networks. Adv. Funct. Mater. 18, 2428-2435 (2008)(a).

Yakacki, C.M., Willis, S., Luders, C., Gall, K.: Deformation limits in shape-memory polymers. Adv. Eng. Mater. 10, 112-119 (2008)(b).

Zhao, Q., Qi, H.J., Xie, T.: Recent progress in shape memory polymer: New behavior, enabling materials, and mechanistic understanding. Prog. Polym. Sci. 49--50, 1-42 (2015).

\begin{tabular}{|l|}
\hline Formatted: Not Highlight \\
\hline Formatted: Not Highlight \\
\hline Formatted: Not Highlight \\
\hline Formatted: Not Highlight \\
\hline
\end{tabular}

